\title{
Planejamento e gestão de recursos hídricos: dificuldades conceituais e de compilação de dados
}

\author{
Planning and management of water resources: conceptual difficulties and of data compilation
}

\author{
Marcio Augusto Reolon Schmidt' ,Marcio Ricardo Salla², Maria Lígia Chuerubim³ ${ }^{3}$,Emiliano Silva Costa ${ }^{4}$ \\ 1,2,3 Faculdade de Engenharia Civil, Universidade Federal de Uberlândia \\ ${ }^{4}$ Mestrando no Programa de Pós-Graduação em Engenharia Civil, Universidade Federal de Uberlândia.
}

\begin{abstract}
Resumo
As ferramentas de auxílio ao planejamento e gestão de recursos hídricos em escala de bacia hidrográfica sofreram um imenso avanço na capacidade de tomada de decisões nas últimas décadas. A interdisciplinaridade para a utilização dessas ferramentas exige conhecimentos conceituais diversos, cujos manuais técnicos são direcionados aos profissionais diretamente envolvidos. Os usuários iniciantes apresentam déficits de conhecimento nas diversas áreas, tais como hidrologia fluvial e geoprocessamento, que é a base de discussão deste artigo. Também foram apresentadas técnicas para compilação e tratamento dos dados de precipitação e quantidade da água superficial, tão importantes como dados de entrada nas modelagens matemáticas de planejamento e gestão de recursos hídricos. Em qualquer estudo de intervenção hídrica em escala de bacia hidrográfica, o usuário deve munir-se de informações históricas que possibilite entender o balanço hídrico e a caracterização da qualidade da água na região de interesse e, a partir daí, definir prognósticos para o uso sustentável dos recursos hídricos. Paralelamente, enfatiza-se que o uso integral das potencialidades de uma ferramenta computacional somente é possível com o entendimento do manual técnico.
\end{abstract}

Palavras-chaves: Planejamento e gestão de recursos hídricos, ferramentas computacionais, compilação de dados, interdisciplinaridade..

\begin{abstract}
The tools to support planning and management of water resources in the river basin scale suffered a huge breakthrough in the ability of decision-making in recent decades. The interdisciplinarity for the use of these tools requires several knowledge conceptual, whose technical manuals are targeted to professionals directly involved. Novice users have deficits knowledge in various areas such as river hydrology and GIS, which is the basis for discussion of this paper. Also were presented techniques for compiling and processing of rainfall data and quantity of surface water, so important as input data for mathematical models for planning and management of water resources. In any study of water intervention in river basin scale, the user must equip historical information that enables understanding the water balance and the characterization of water quality in the region and, from there, set predictions for sustainable use of water resources. At the same time, it is emphasized that the full use of the potential of a computational tool is possible only with the understanding of the technical manual.
\end{abstract}

Keywords: Planning and management of water resources, computational tools, data compilation, interdisciplinarity. 


\section{INTRODUÇÃO}

As ferramentas de auxílio ao planejamento e gestão de recursos hídricos em escala de bacia hidrográfica sofreram um imenso avanço na capacidade de tomada de decisão nos últimos tempos. Em todo o mundo existem muitas ferramentas computacionais, com complexidades matemáticas distintas e úteis à gestão de múltiplos usos da água, tais como Wargi-Sim (SECHI \& SULIS, 2009), HecRas (FAN et al., 2009), Aquatool (PAREDES-ARQUIOLA et al., 2010), , Modflow (XU et al., 2012), Topmodel (CHEN \& WU, 2012), entre outros.

O comprometimento na qualidade da água superficial impulsionou o surgimento de diversos modelos de qualidade da água, com níveis distintos de complexidade matemática (SALLA, 2013). Atualmente, os modelos com maior aceitabilidade são Qual2K (CHAPRA et al., 2008), Gescal (PAREDES-ARQUIOLA et al., 2010), Aquatox (MAMAQANI et al., 2011), Mike (DOULGERIS et al., 2012), Wasp (YENILMEZ \& AKSOY, 2013) e Swat (BALATHANDAYUTHAM et al., 2014). As referências informadas anteriormente são de alguns estudos recentes com o uso dos modelos.

A implantação de planos de recursos hídricos em escala de bacia hidrográfica trouxe consigo a utilização de ferramentas de Sistema de Informação Geográfica (SIG) aos modelos de planejamento e gestão ambientais, com destaque para trabalhos desenvolvidos por Zhang et al. (2011) e Sulis \& Sechi (2013) e Bhatt et al. (2014).

Atuam profissionais de diversas áreas do conhecimento na implementação e aplicação das ferramentas suportes à decisão. Focando-se no usuário iniciante, seja aluno de iniciação científica, mestrado, profissional ou recém-formado, a enorme interdisciplinaridade pode dificultar o manuseio correto das ferramentas. Como exemplos, os engenheiros civis possuem conhecimentos limitados sobre ferramentas de geoprocessamento e suas aplicações em recursos hídricos, já os geógrafos não apresentam conhecimentos mínimos de hidráulica fluvial em escala de bacia hidrográfica. São frequentes e numerosos os questionamentos de ordem conceitual feitos pelos alunos de diversas áreas, consequência da inexistência de tais assuntos nas grades curriculares dos referidos cursos de graduação.

O objetivo deste artigo é auxiliar estes usuários, principalmente de regiões nas quais existem limitações de dados quantitativos monitorados, na utilização de ferramentas computacionais de suportes à decisão em recursos hídricos.

\section{METODOLOGIA}

O contato constante dos autores deste artigo com discentes de graduação e pós-graduação da área de recursos hídricos evidencia as reais dificuldades conceituais e de compilação de dados no manuseio de ferramentas computacionais de suporte à decisão.

Nesta perspectiva, são mencionados e discutidos conceitualmente temas que geram enormes dificuldades, tais como hidrologia fluvial e geoprocessamento. São apresentadas algumas metodologias para correta compilação de dados de entrada requeridos pelas ferramentas de gestão integrada de recursos hídricos.

\section{RESULTADOS E DISCUSSÃO}

A compreensão conceitual de manuais técnicos de ferramentas computacionais, juntamente com a percepção na seleção de dados mínimos necessários à modelagem traz a discussão que segue.

\subsection{Hidrologia fluvial}

Aqui é dado enfoque à vazão superficial, que é a principal variável envolvida na modelagem quantitativa e, consequentemente, na modelagem qualitativa em cursos de água naturais. Dentro da modelagem de qualidade da água em rios, a vazão superficial ou precipitação efetiva é a que possui a maior importância devido à sua elevada velocidade de transporte e diluição das substâncias poluentes.

Em função da ausência de postos de monitoramento de vazão em bacias hidrográficas com elevado consumo de água, torna-se indispensável o conhecimento de metodologias para determinação da 
vazão superficial em campo, transferência espacial de informações de vazão entre bacias hidrográficas e estabelecimento de relações hidráulicas, além do conhecimento da importância da vazão de referência.

\section{Vazão superficial em campo}

Principalmente em regiões com elevada densidade demográfica, a falta de dados de vazão superficial é um empecilho ao adequado planejamento e gerenciamento dos recursos hídricos. No Brasil, devido ao alto custo de implantação e manutenção, existem deficiências de dados fluviométricos históricos (SALLA et al., 2013).

Em uma estação fluviométrica, indiretamente, a vazão é obtida por meio da relação entre o nível líquido na seção transversal e sua correspondente vazão, relação esta chamada curva-chave. A estação fluviométrica é uma instalação hidráulica, situada às margens da seção transversal de interesse, onde são instaladas réguas linimétricas ou linígrafo para medição da variação temporal do nível líquido.

De acordo com Von Sperling (2007), na escolha da seção transversal deve-se considerar alguns aspectos, tais como: facilidade de acesso, proximidade com uma unidade de estudo que garanta constante supervisão, geometria da seção transversal bem definida e trecho de rio retilíneo com escoamento bem tranquilo (preferencialmente com número de Froude inferior à 1).

Na prática, o método área-velocidade é o mais utilizado na determinação da vazão. Neste método simplesmente multiplica-se a velocidade média do escoamento pela área da seção transversal do curso de água natural. De acordo com Salla et al. (2013), o traçado da seção transversal pode ser obtido com equipamentos específicos, como teodolito e ecobatímetro.

Adicionalmente, a velocidade média do escoamento pode ser determinada por medições diversas e pontuais com equipamento molinete ou pela medição instantânea da varredura da seção transversal com o equipamento Acoustic Doppler Current Profiler (ADCP). Todavia, a escolha do equipamento está diretamente relacionada com a disponibilidade financeira e técnica. Apesar das limitações práticas quanto à utilização do equipamento molinete em cursos de água com elevada seção transversal, ainda assim é muito utilizado em regiões com poucos recursos financeiros.

Desta forma, se por um lado o equipamento molinete é mais barato e não necessita essencialmente de mão de obra especializada, por outro, o equipamento ADCP, além de apresentar um alto custo, deve ser utilizado por uma equipe técnica previamente treinada. Além disso, os resultados alcançados com a utilização do ADCP são mais precisos.

\section{Vazão de referência}

A vazão de referência é definida a partir do objetivo da modelagem hidrológica ou de qualidade da água (VON SPERLING, 2007). Considera-se:

- Escala diária ou mensal da vazão média, em um determinado período, para calibrar e validar os coeficientes integrantes das formulações matemáticas;

- A vazão média anual ou vazão média em meses chuvosos ou em meses de seca para simular uma situação específica;

- A vazão máxima é muito utilizada na previsão de ocorrência de enchente em áreas urbanas;

- A vazão mínima é utilizada no planejamento dos recursos hídricos, para definir a liberação ou não de outorga para captação de água e de lançamento de efluente tratado e para determinar processos e eficiências mínimas de tratamento em estação depuradora. Para isso realiza-se o estudo da capacidade de autodepuração do curso de água diante da situação mais crítica possível, em que a capacidade de transporte e diluição de poluentes é mínima.

A capacidade de autodepuração é avaliada a partir da conformidade ou não dos parâmetros ambientais com os valores limites definidos pelos órgãos governamentais. No Brasil, as Resoluções do Conselho Nacional de Meio Ambiente - CONAMA 357:2005 e 430:2011 são, respectivamente, responsáveis pela classificação dos cursos de água no território nacional e pelas condições e padrões de lançamento de efluente. 
As vazões mínimas de referência mais utilizadas no planejamento de recursos hídricos no Brasil são $\mathrm{Q}_{90}, \mathrm{Q}_{95}$ e $\mathrm{Q}_{7,10}$. As vazões de referência $\mathrm{Q} 90$ e $\mathrm{Q} 95$ são, respectivamente, as vazões nas quais $90 \%$ e $95 \%$ dos dados históricos são iguais ou superiores a ela. Já a vazão de referência $Q_{7,10}$ compreende a vazão mínima, em 7 dias consecutivos, para um período de 10 anos de dados históricos. As metodologias de cálculo destas vazões de referência são apresentadas detalhadamente em Von Sperling (2007) e Chapra (2008).

\section{Transferência espacial de informações de vazão}

O território nacional é deficitário na quantidade de estações fluviométricas em operação, onde as prioridades situam-se em outros setores da administração pública. Isso faz com que a transferência espacial de informações de vazão em escala de bacia hidrográfica seja vista como uma metodologia muito utilizada para estimar a vazão em regiões com carências de monitoramento. Os métodos mais simples e utilizados são:

\section{- descarga específica}

A maneira mais simples, porém menos precisa, de associar dados quantitativos entre bacias hidrográficas distintas é por meio da descarga específica, geralmente em $\mathrm{hm}^{3} \cdot \mathrm{mês}^{-1} \cdot \mathrm{km}^{-2} \mathrm{ou} \mathrm{m}^{3} \cdot \mathrm{s}^{-1} \cdot \mathrm{km}^{-2}$. Este procedimento deve ser aplicado em bacias hidrográficas que possuem características similares quanto ao clima, cobertura do solo, tipo de solo, topografia, entre outras. Existem duas possibilidades para a regionalização da vazão, que depende da localização da estação fluviométrica. Na primeira possibilidade, a estação fluviométrica situa-se na foz de uma sub-bacia vizinha, enquanto que, na segunda possibilidade, a estação fluviométrica situa-se na foz da bacia hidrográfica cuja sub-bacia sem dados está inserida.

Primeiramente, deve-se calcular a vazão específica na estação fluviométrica com dados históri$\cos \left(\mathrm{em} \mathrm{hm^{3 }} \cdot \mathrm{mês}^{-1} \cdot \mathrm{km}^{-2}\right.$ ou m $\left.\mathrm{m}^{3} \cdot \mathrm{s}^{-1} \cdot \mathrm{km}^{-2}\right)$. Na sequência, a vazão em outra bacia hidrográfica sem dados de referência é estimada por meio da multiplicação de sua área de drenagem (em $\mathrm{km}^{2}$ ) pelo valor da vazão específica.

\section{- regionalização por curva de probabilidade}

É utilizada para estimar a vazão mínima, máxima e média de longo período em regiões onde não existem estações de monitoramento. A aplicabilidade dessas três vazões no planejamento e gestão de recursos hídricos foi informada no sub-item Vazão de referência. De acordo com Hidrotec (2014), a sequência para regionalização da curva de probabilidade é:

- Levantamento de dados hidrológicos e físicos das bacias hidrográficas envolvidas;

- Cálculo das precipitações médias nas sub-bacias de contribuição. O item 3.3 traz alguns métodos;

- Escolha de sub-bacias homogêneas hidrologicamente, levando-se em consideração similaridades no uso e ocupação do solo, tipo de solo e cobertura vegetal, clima, usos múltiplos da água, entre outros;

- Escolha de metodologia para determinação da vazão de interesse, seja mínima, máxima e média de longo prazo, disponíveis em Hidrotec (2014) e Tucci (2004).

- regionalização da curva de permanência

A curva de permanência é utilizada para regionalização de vazão quando o foco é saber a amplitude de variação ou frequência com que o valor da vazão é excedido em qualquer ponto dentro da bacia hidrográfica. Nesta situação, o usuário não tem nenhum interesse na quantificação de vazão mínima, máxima ou média de longo período. A metodologia para determinação da curva de permanência pode ser encontrada em Hidrotec (2014) e Tucci (2004).

\section{- regionalização da curva de regularização}

Em muitas regiões do Brasil existe enorme variação mensal da vazão nos cursos de água naturais, onde sobra água nos períodos chuvosos e falta água nos períodos de estiagem. Em cidades brasileiras de médio e grande porte, em função dos períodos acentuados de seca, a melhor solução 
para garantir as demandas de irrigação, abastecimento público, geração de energia elétrica, navegação fluvial, dentre outros, é por meio de reservatório de regularização.

Uma curva de regularização relaciona o volume armazenado com a vazão média de longo prazo, para uma determinada probabilidade de ocorrência. A partir de relações adimensionais do volume regularizado e da vazão com a vazão média de longo período é possível ajustar uma função para uma determinada probabilidade de ocorrência. Esta função pode ser regionalizada para uma bacia hidrográfica vizinha com características físicas semelhantes. Tucci (2004) permite uma leitura mais aprofundada sobre o tema.

\section{Relações hidráulicas}

A modelagem de qualidade da água, realizada por meio de ferramentas computacionais, possibilita que um trecho de curso da água seja discretizado em vários subtrechos, cujas características hidráulicas podem assumir valores diferentes em função das variações geométricas na seção transversal, na declividade e no tipo de material de fundo e do talude. Para a maioria das ferramentas existentes atualmente, o escoamento nos subtrechos é considerado permanente e uniforme, no qual as relações hidráulicas da velocidade média v, do nível líquido h e da largura de superfície L em função da vazão $\mathrm{Q}$ podem ser obtidas pela equação de Manning ou por relações potenciais. Independente da equação utilizada o usuário deve, inicialmente, para cada trecho ou subtrecho, definir a forma geométrica da seção transversal. Usualmente, em regiões onde não existentes dados de campo, utiliza-se a forma trapezoidal com inclinações dos taludes similares.

Com relação à equação de Manning, os dados de entrada são: coeficiente de rugosidade de Manning $\eta\left(\mathrm{m}^{-1 / 3} \mathrm{~s}\right)$, largura $\mathrm{b}$ da base do canal $(\mathrm{m})$, declividade de fundo $\mathrm{I}(\mathrm{m} / \mathrm{m})$ e declividade $\alpha$ dos taludes (graus). A partir dos dados de entrada, as ferramentas computacionais relacionam internamente o nível líquido $\mathrm{h}$, a velocidade média v e a largura da superfície $\mathrm{L}$ com a vazão Q. Iterativamente, a profundidade média h é obtida diante da igualdade da função do escoamento (que depende da vazão $\mathrm{Q}$, da declividade de fundo I e da rugosidade das paredes $\eta$ ) com a função geométrica da seção transversal (que depende da largura da base $b$, declividade do talude $\alpha$ e da profundidade média $h$ ), cuja formulação matemática está ilustrada na Equação 1.

(Q. $\eta) / I^{1 / 2}=\left[\left(b \cdot h+h^{2} \cdot \operatorname{cotg} \alpha\right)^{5 / 3}\right] /\left\{b+2 \cdot\left[h^{2} \cdot(\operatorname{cotg} \alpha+1)^{1 / 2}\right]\right\}^{2 / 3}$

Equação 1

Na prática, em função da baixa declividade longitudinal existente em um curso da água natural, estima-se a declividade de fundo como a diferença entre a cota topográfica inicial e final do trecho dividido pela distância longitudinal. Já as rugosidades das paredes são tabeladas e podem ser consultadas em Von Sperling (2007), Vianna (2007), Netto et al. (1998), entre outros.

A relação da largura de superfície L com a vazão Q é obtida pela Equação 2. E, de acordo com a equação da continuidade, a relação da velocidade média v com a vazão $Q$ é obtida por meio da Equação 3.

$\mathrm{L}_{(\mathrm{Q})}=\mathrm{b}+2 \cdot \mathrm{h}_{(\mathrm{Q})} \cdot \operatorname{cotg} \alpha$

Equação 2

$\mathrm{v}_{(\mathrm{Q})}=\mathrm{Q} /\left(\mathrm{b} \cdot \mathrm{h}_{(\mathrm{Q})}+\mathrm{h}_{(\mathrm{Q})}^{2} \cdot \operatorname{cotg} \alpha\right)$

Equação 3

No tocante às relações potenciais, as mesmas podem ser utilizadas somente quando, em um determinado trecho de rio, existem dados históricos de nível líquido $h$. A relação potencial $h=\alpha 1$. $Q^{\beta 1}$ pode ser obtida diretamente na planilha de cálculo Excel, na qual as constantes $\alpha 1$ e $\beta 1$ são ajustadas por linha de tendência potencial ou por outro método de otimização (por exemplo, Solver). As relações hidráulicas entre a vazão média $\mathrm{Q}$ com a velocidade média $\mathrm{v}\left(\mathrm{v}=\alpha 2 \cdot \mathrm{Q}^{\beta 2}\right)$ e com a largura de superfície $\mathrm{L}\left(\mathrm{L}=\alpha 3 . \mathrm{Q}^{\beta 3}\right)$ são obtidas da mesma forma, em função dos pares de valores $v, \mathrm{Q}$ e $\mathrm{L}, \mathrm{Q}$, calculados por meio das Equações 2 e 3, respectivamente. 


\subsection{Geoprocessamento}

Geoprocessamento pode ser entendido como um termo guarda-chuva, sob o qual estão reunidas diversas tecnologias de coleta e processamento de dados referenciados a posições sobre a superfície da Terra. Essas tecnologias envolvem técnicas de coleta de dados como os sistemas de posicionamento por satélites artificiais (GNSS - Global Navigation Satellite System), Sensoriamento Remoto e de análise e processamento como os Sistemas de Informação Geográfica e Banco de Dados Geográficos (BDG).

De acordo com Câmara et al. (2001), geoprocessamento é uma tecnologia interdisciplinar, que permite a convergência de diferentes disciplinas científicas para o estudo de fenômenos ambientais e urbanos, auxiliando o processo de tomada de decisões, que corresponde, na prática, a um conjunto de geotecnologias que utilizam uma "linguagem comum" para as diferentes áreas do conhecimento como, por exemplo, nos cursos de Engenharia Ambiental, Engenharia Civil, Arquitetura e Geografia, Agronomia, Zootecnia, dentre outros.

Para a análise de fenômenos como o comportamento do relevo e do uso e ocupação do solo, ferramentas como o Sistema de Informação Geográfica - SIG associam dados espaciais, atributos e algoritmos de análise espacial, de forma a permitir uma análise ampla e complexa da região de estudo, evidenciando padrões dos dados espaciais coletados na bacia hidrográfica, como fatores de qualidade da água, vazão, entre outros, de maneira sintética e integrada.

Essas análises espaciais se desenvolvem com a modelagem dos dados e simulação de cenários para servirem como subsídio à elaboração de alternativas para a análise de uso e ocupação do solo, ordenamento territorial, equipamentos urbanos e monitoramento ambiental, entre outras aplicações complexas, que envolvem diferentes componentes dinâmicos (MOTA,1999).

Ao permitir a integração de dados espaciais e não espaciais e pela sua capacidade de realizar análises topológicas e lógicas baseadas nesses dados geométricos e de atributos, respectivamente, o SIG integra informações de dados cartográficos, cadastrais de diferentes naturezas, variáveis ambientais, entre outras, em um banco de dados unificado, o que reflete a multiplicidade de usos e a interdisciplinaridade permitida.

Entretanto, muitos desafios estão associados com a integração de SIG com modelos hidrológicos. Exemplos recentes são os trabalhos de Mckinneya e Cai (2002), Ferrero (2004), Hansen (2008) e HecRas (HEC, 2014). Estes autores afirmam que a associação das tecnologias de geoprocessamento a modelos hidrológicos representam um grande potencial e avanço na qualidade dos estudos e análises dos fenomenos. Por outro lado, é exigido um profissional multidisciplinar que consiga transitar entre as diferentes áreas e que consiga interpretar o problema de forma adequada, selecionar as melhores configurações para coleta e processamento dos dados espaciais e de água e selecionar os métodos de análise mais adequados.

\subsection{Compilação dos dados}

As ferramentas de suporte à decisão em recursos hídricos podem ou não trazer restrições quanto às séries temporais dos dados de entrada. Muitas dessas ferramentas foram desenvolvidas, inicialmente, para aplicações em países localizados no hemisfério Norte e consideram os dados de entrada a partir do ano hidrológico, que possui como data inicial o primeiro dia da estação chuvosa e como data final o último dia da estação seca.

No processo de modelagem quantitativa e qualitativa da água, a etapa mais trabalhosa e mais demorada refere-se à coleta e organização dos dados de entrada, uma vez que exige a participação direta de outros profissionais da área de recursos hídricos. Nesta fase, o usuário necessita de muitos dados, coletados em locais distintos e em diferentes épocas, o que conduz a necessidade de buscar informações e/ou dados em diferentes setores da sociedade civil, como em órgãos privados e públicos, tais como autarquias de água e esgoto municipais e estaduais, empresas responsáveis pelo gerenciamento de centrais hidrelétricas, comitês de bacias hidrográficas, instituições de ensino e pesquisa e órgãos estaduais e nacionais relacionados com a área ambiental.

Transcorrido o período de coleta dos dados, inicia-se a etapa, não menos trabalhosa, de organização desses dados. O usuário deve se preocupar com a possibilidade de ausência, repetição ou duplicação dos dados e, principalmente, com sua consistência regional. Estas verificações demandam uma quantidade de tempo considerável em todo o processo de modelagem. Neste contexto, seguem algumas recomendações: 
- Devido à importância dos dados de qualidade da água na calibração e validação do modelo, deve-se certificar sobre sua procedência;

- Os dados de vazão e nível líquido faltantes podem ser estimados. Para isso, quando a simulação é feita em escala mensal, por exemplo, a estimativa para um mês faltante pode ser obtida pela média dos valores deste mês em anos diferentes e dentro do período de simulação. O problema da duplicidade ou repetição de dado deve ser tratado com cautela, no qual o órgão responsável pelo fornecimento deste dado deve ser consultado para melhores esclarecimentos sobre o ocorrido;

- A ausência ou falha de registro de precipitação, em períodos variados, pode ser preenchida por diversos métodos estatísticos, tais como método de ponderação regional, método de regressão linear e método de ponderação regional com base em regressões lineares. Em função da facilidade no manuseio e aos bons resultados, o método de regressão linear apresenta elevada aceitação, o qual correlaciona os dados falhos de precipitação de um posto de monitoramento com dados existentes de um posto vizinho por meio de equação linear;

- É necessário também analisar a consistência dos dados de precipitação de um posto de monitoramento frente aos dados de um posto vizinho (ou seja, verificar a homogeneidade regional). Os dois métodos mais conhecidos são: método da dupla massa e método do vetor regional;

- Os dados de entrada de precipitação em uma bacia hidrográfica são obtidos a partir de estações pluviométricas existentes dentro da bacia em estudo e de sua vizinhança mais próxima. Estes dados podem ser calculados de duas maneiras. Na primeira, estima-se a precipitação média na bacia hidrográfica considerando-se uma lâmina de água uniforme em toda a área para um determinado período de tempo, cujos métodos mais usuais e com distintas peculiaridades são: método da média aritmética, método de Thiessen e método das isoietas. Já na segunda, estimam-se os dados de precipitação em um único ponto na bacia hidrográfica, que pode ser o centróide ou um outro ponto representativo, a partir dos dados existentes em estações pluviométricas vizinhas. Para isso, são frequentemente utilizados os modelos determinísticos locais, cuja representação matemática da equação de interpolação está ilustrada na Equação 4.

$$
P_{i}=\sum_{j=1}^{n} f_{i j} \cdot P_{j} / \sum_{j=1}^{n} f_{i j}
$$

Na qual: $\mathrm{P}_{\mathrm{i}}$ refere-se ao o valor da precipitação no centróide ou em outro ponto representativo i; $\mathrm{P}_{\mathrm{j}}$ compreende a precipitação em uma estação pluviométrica vizinha $\mathrm{j}$ e fij é um fator de ponderação. As alternativas com relação aos interpoladores são: vizinho mais próximo, média simples e média ponderada.

A interpolação por vizinho mais próximo é pouco utilizada, pois considera que o valor da precipitação no centróide ou em um ponto representativo na bacia hidrográfica é igual ao valor da estação pluviométrica mais próxima, sem interpolá-lo. A interpolação por média simples considera que o valor da precipitação no centróide ou ponto representativo é igual à média simples dos valores encontrados nas estações mais próximas.

Neste caso, o fator de ponderação $\mathrm{f}_{\mathrm{ij}}$ assume valor igual a $1 / \mathrm{n}$, sendo $\mathrm{n}$ o número de estações pluviométricas vizinhas. A interpolação por média ponderada é a mais utilizada, na qual o fator de ponderação $\mathrm{f}_{\mathrm{ij}}$ assume valor igual a $1 / \mathrm{d}_{\mathrm{ij}}{ }^{2}$, denominado como inverso do quadrado da distância. $\mathrm{O}$ termo $d_{i j}$ é a distância entre o centróide ou ponto representativo i e a estação pluviométrica vizinha ${ }_{j}$, obtida por meio de plataforma SIG.

Existem outras funções de ponderação mais complexas e realísticas. Com exemplo, têm-se os estimadores de densidade não paramétricos ou Kernel estimators, que generalizam o princípio de média móvel local produzindo dados mais suaves. Outros métodos de interpolação mais sofisticados devem ser consultados na literatura da área;

- A modelagem de qualidade da água em rio pode ser dividida em diversos subtrechos no curso de água, definidos a partir da variabilidade das entradas, demandas e estações fluviométrica e de qualidade da água. Normalmente, dados de nível líquido estão vinculados aos de vazão em uma seção transversal. Isso faz com que o usuário iniciante depare-se com um dilema, que é: Devo considerar as relações hidráulicas potenciais no subtrecho a montante dessa seção transversal, no sub-trecho a 
jusante ou em ambos os subtrechos? Normalmente, nesta situação, considera-se a metade da distância do trecho de montante mais a metade da distância do trecho de jusante para as mesmas características hidráulicas potenciais;

- Frequentemente, em bacia hidrográfica com elevada disponibilidade hídrica superficial, a variabilidade da vazão transportada ao longo do rio principal faz com que seja inviável o monitoramento de nível líquido no médio e baixo curso de água (em função dos elevados níveis líquidos). Com isso, é usual utilizar as relações hidráulicas potenciais no alto curso de água e a equação de Manning no médio e baixo curso de água;

- As ferramentas computacionais permitem que as demandas superficiais sejam introduzidas de forma fixa ou variável ao longo do ano. As demandas outorgadas para o consumo humano, usos industriais e irrigação são introduzidas de forma fixa (ou seja, valores fixos em todos os meses do ano). $\mathrm{Na}$ existência de central hidrelétrica, a demanda turbinada deve ser introduzida de forma variável.

\section{CONCLUSÕES}

Em qualquer estudo de intervenção hídrica em escala de bacia hidrográfica, o usuário deve munir-se de informações históricas que possibilite entender o balanço hídrico e a caracterização da qualidade da água na região de interesse e, a partir daí, definir prognósticos para o uso sustentável dos recursos hídricos. Paralelamente, enfatiza-se que o uso integral das potencialidades de uma ferramenta computacional somente é possível com o entendimento do manual técnico.

\section{REFERÊNCIAS}

BALATHANDAYUTHAM, K.; MAYILSWAMI, C. A semi-distributed water balance model for Walayar sub basin using SWAT model, International Journal of Agricultural Engineering, vol. 7(1), p. 198-202, 2014.

BHATT, G.; KUMAR, M.; DUFFY, C.J. A tightly coupled GIS and distributed hydrologic modeling framework. Environmental Modelling \& Software. vol. 62, p. 70-84, december, 2014.

BRASIL. CONSELHO NACIONAL DO MEIO AMBIENTE. Resolução nº 357, de 17 de março de 2005. Disponível em: http://www.mma.gov.br/port/conama/legiabre.cfm?codlegi=459. Acesso em: 13 jun. 2014.

BRASIL. CONSELHO NACIONAL DO MEIO AMBIENTE. Resolução nº 430, de 13 de maio de 2011. Disponível em: http://www.mma.gov.br/port/conama/legiabre.cfm?codlegi=646. Acesso em: 13 jun. 2014.

CÂMARA, G., DAVIS, C.; MONTEIRO, A. M.V. Introdução à Ciência da Geoinformação. Instituto de Pesquisas Espaciais (INPE), São José dos Campos, 2001. Disponível em: http://www.dpi.inpe.br/gilberto/livro/ introd/. Acesso em: 13 jun. 2014.

CHAPRA, S.C., G.J. PELLETIER \& H. TAO. QUAL2K: A Modeling Framework for Simulating River and Stream Water Quality, Version 2.11: Documentation and Users Manual. Civil and Environmental Engineering Dept., Tufts University, Medford, MA, 109 pp, 2008. Disponível em: http://epa.gov/athens/wwqtsc/html/ qual2k.html. Acesso em: 13 jun. 2014.

CHEN, J.; WU Y. Advancing representation of hydrologic processes in the Soil and Water Assessment Tool (SWAT) through integration of the TOPographic MODEL (TOPMODEL) features. Journal of Hydrology, vol. 420-421, p. 319-328, february, 2012.

DOULGERIS, C.; GEORGIOU, P.; PAPADIMOS, D.; PAPAMICHAIL, D. Ecosystem approach to water resources management using the MIKE 11 modeling system in the Strymonas River and Lake Kerkini. Journal of Environmental Management, vol. 94, n. 1, p. 132-143, february, 2012. 
FAN, C.; KO, C.H.; WANG, W.S. An innovative modeling approach using Qual2K and HEC-RAS integration to assess the impact of tidal effect on river Water quality simulation. Journal of Environmental Management, vol. 90, n. 5, p. 1824-1832, april, 2009.

FERRERO, V. O. Hidrología computacional y modelos del terreno. 2004. Disponível em: <http://www. gabrielortiz.com/descargas/Hidrologia_Computacional_MDT_SIG.pdf>. Acesso em: 11 mai 2014.

HANSEN, M. A. F. As práticas de campo em eventos de inundação com o apoio de informações espaciais. 2008. Disponível em: <http://www.inpe.br/crs/crectealc/pdf/hansen.pdf>. Acesso em:14 mar 2014.

HEC - Hydrologic Engineering Center. US Army Corps of Engineers.Davis, USA: Institute for Water Resources, 2014. Disponível em: <http://www.hec.usace.army.mil/>. Acesso em: 08 jul 2014.

HIDROTEC - Geração e transferência de tecnologia em recursos hídricos para o Estado de Minas Gerais. Universidade Federal de Viçosa, Viçosa. Disponível em: http://www.hidrotec.ufv.br. Acesso em: 28 de nov. 2014.

MAMAQANI, A; KHORASANI, N.; TALEBI K.; HASHEMI, S.H.; RAFIEE G.; KHOSROSHAHI, F.B. Diazinon fate and toxicity in the tajan River (Iran) ecosystem. Environmental Engineering Science, vol. 28, p. 1-10, 2011.

MCKINNEYA, D. C. e CAI, X.. Linking GIS and water resources management models: an object-oriented method. Environmental Modelling \& Software 17 (2002) 413-425

MOTA, S. Preservaçãoeconservaçãode recursoshídricos.2.ed.RiodeJaneiro:ABES, 1999.

MONICO, J. F. G. Posicionamento pelo GNSS: descrição, fundamentos e aplicações. $2^{\mathrm{a}}$ Edição. São Paulo: UNESP, 476p., 2008.

NETTO, A., FERNANDEZ, M.F., ARAUJO, R., ITO, A.E. Manual de Hidráulica. $8^{a}$ edição atualizada. São Paulo: Edgard Blücher, 670p., 1998.

PAREDES-ARQUIOLA, J.; ÁLVAREZ, J.A.; SOLERA, A. A decision support system for water quality issues in the Manzanares River (Madrid, Spain). Science of the Total Environment, vol. 408, n. 12, p. 2576$2589,2010$.

SALLA, M.R., PEREIRA, C.E., ALAMY-FILHO, J.E., DE PAULA, L.M., PINHEIRO, A.M. Estudo da autodepuração do Rio Jordão, localizado na bacia hidrográfica do Rio Dourados. Revista Engenharia Sanitária e Ambiental, vol. 18, n. 2, p. 105-114, 2013.

SECHI, G.M.; SULIS, A. Water system management through a mixed optimization-simulation aproach, Journal Water Resource Pl-Asce, vol. 135, n. 3, p. 160-170, 2009.

SULIS, A.; SECHI, G.M. Comparison of generic simulation models for water resource systems, Environmental Modelling \& Software, vol. 40, p. 214-225, february, 2013.

TUCCI, C. E. M. Hidrologia - Ciência e Aplicação. $3^{a}$ edição. Porto Alegre: UFRGS Editora, 943p., 2004.

VALENTE, J. A. Por quê o Computador na Educação? Campinas, SP: Gráfica Central da UNICAMP, 1993. Disponível em: http://www.ich.pucminas.br/pged/db/wq/wq1_LE/local/txtie9doc.pdf. Acesso em: 13 jun. 2014.

VIANNA, M.R. Hidráulica para engenheiros sanitaristas e ambientais. volume 1: Fundamentos. FUMEN/ FEA, $1^{\text {a }}$ edição, 2007. 
VON SPERLING, M. Estudos e modelagem da qualidade da água de rios. Departamento de Engenharia Sanitária e Ambiental, Universidade Federal de Minas Gerais, Belo Horizonte, Ed. UFMG, vol.7, 588 p, 2007.

XU, X.; HUANG, G.; ZHAN H.; QU, Z.; HUANG Q. Integration of SWAP and MODFLOW-2000 for modeling groundwater dynamics in shallow water table areas. Journal of Hydrology, vol. 412-413, p. 170-181, january, 2012.

YENILMEZ, F.; AKSOY, A. Comparison of phosphorus reduction alternatives in control of nutrient concentrations in Lake Uluabat (Bursa, Turkey): Partial versus full sediment dredging. Limnologica, vol. 43, p. 1-9, 2013.

ZHANG, X.; HUANG, G.H.; NIE, X.; LI. Q. Model-based decision support system for water quality management under hybrid uncertainty. Expert Systems with Applications, vol. 38, n. 3, p. 2809-2816, march, 2011. 